\title{
For the Healthcare Provider: Recognition of Heart Disease
}

\author{
LoriAnn F. Pajalich, DNP, RN, CNS, GCNS-BC \\ Assistant Professor of Nursing, Passan School of Nursing at Wilkes \\ University, N218 - Stark Learning Center,USA.
}

\author{
*Corresponding author \\ LoriAnn F. Pajalich, Assistant Professor of Nursing, Passan School of \\ Nursing at Wilkes University , N218 - Stark Learning Center,USA,Tel \\ :+ 570.408.4076; E-Mail: loriann.pajalich@wilkes.edu.
}

Submitted: 03 May 2017; Accepted: 06 May 2017; Published: 10 Aug 2017
Mr. and Mrs. Jones just arrived home from celebrating their $35^{\text {th }}$ wedding anniversary with a family dinner. Mrs. Jones, a 56 year old female, begins to complain of nausea, back pain, cold sweats, and feeling dizzy. She had some anxiety, but brushes it off as the start of "something." Her husband reminds her that she has been complaining of not feeling well for the last few weeks and suggests that they drive to the local emergency room for an antibiotic.

Every 43 seconds, someone in the United States has a myocardial infarction, or a heart attack [1]. This type of acute coronary syndrome occurs when a part of the heart muscle doesn't receive enough blood flow. The more time that passes without treatment to restore blood flow, the greater the damage to the heart muscle.

So what is an acute coronary syndrome? It is an umbrella term used to cover a group of signs and symptoms of chest pain or acute myocardial ischemia due to an insufficient blood supply to the heart muscle that results from clogged arteries. Clinical symptoms are classified as either unstable angina, stable angina, a non $Q$ wave myocardial infarction (NONSTEMI), or an acute myocardial infarction (STEMI).

Unstable angina is also referred to as preinfarction angina or crescendo angina. Symptoms with this type of angina produce:

- An increase in frequency and severity

- Occurs often at rest, while sleeping, or with little exertion

- Unexpected

- $\quad$ Severe and lasts as long as 30 minutes

- Usually not relieved with rest or medications

- May continually get worse

- May signal a myocardial infarction may occur

Stable angina signs and symptoms include:

- An occurrence when the heart muscle is working harder

- $\quad$ Expected, predictable, and episodes are alike

- May last 5 minutes or less and is relieved with rest or medication

- May feel like gas or ingestion

- Described as chest pain that may radiate to arms, back, and other areas

Non Q Wave Myocardial Infarction (NONSTEMI) is created from a plaque rupture and coronary thrombus that compromise blood flow to the region of the myocardium.
Acute Myocardial Infarction (STEMI) is the necrosis of region of the myocardium secondary to interruption of blood flow to that region of the myocardium.

Coronary heart disease (CHD) is the leading cause of death in women [2]. There are the chief risk factors that predict CHD onset in healthy women are age, low-density lipoprotein (LDL) and highdensity lipoprotein (HDL) cholesterol levels, high blood pressure, diabetes mellitus, and smoking. However, there is evidence that suggests that sex shows a positive correlation to $\mathrm{CHD}$.

Far too often woman ignore their atypical symptoms of cardiac issues because they are not as easily recognized; as do many healthcare providers. According to Davis (2011), the majority of studies have found that women take longer than men to seek care for acutecoronarysyndromes (ACS) symptoms. Women are more likely to have atypical symptoms, perceive themselves as invulnerable to heart disease, and keep their symptoms to themselves. The movie portrays a heart attack as a male who has significant trouble breathing, extreme diaphoresis, and mid sternal pain that radiates to the jaw and arm. Woman experience milder symptoms that are many times under recognized by a healthcare provider or believed to be anxiety related. According to The Woman's Heart Foundation, about a third of women experience no chest pain at all when experiencing a heart attack and $71 \%$ of women report flu-like symptoms for two weeks to a month preceding more acute chest discomfort or severe shortness of breath [3].

Racial and ethnic disparities awareness an important risk factor to understand as well. According to Heron (2012), heart disease is the leading causeof death for African American and Caucasian women in the United States [4]. The higher CVD mortality rate and increased incidence of risk factors among certain racial and ethnic groups, such as hypertension in African American women and diabetes mellitus among Hispanic women lends the push for this increased awareness for healthcare providers. Among Hispanic women, heart disease and cancer cause roughly the same number of deaths each year. For American Indian or Alaska Native and Asian or Pacific Islander women, heart disease is second only to cancer.

Furthermore, age as a risk factor is a significant predictor of risk for $\mathrm{CHD}$ as well. So healthcare providers need to consider racial/ 
ethnic difference and age distribution between populations into account as well. Much of this lack of risk factor stratification is also widespread among patients. Healthcare providers need to target heart disease educations to not only the healthcare community, but to racial/ethnic minorities and women. An emphasis should be placed on the continuum of signs of CHD and heart attacks within these populations.

It is imperative for a healthcare provider to promptly recognize these atypical symptoms of a potential acute coronary syndrome in a woman. Healthcare providers must also keep in mind that some women experience several symptoms while others show all symptoms. In addition, there are women who exhibit no symptoms prior to a myocardial infarction. The earlier a healthcare provider can recognize symptoms of acute coronary syndromes in woman and intervene with appropriate action, the better. Healthcare providers need to identify women who are at high risk for coronary events. These women requires a greater use of secondary prevention treatments for them as well.

\section{Primary Risk Factors and Secondary Risk Factors}

There are modifiable and non-modifiable risk factors that contribute to the development of a cardiac event in an individual. A nonmodifiable risk factor is an occurrence over which a person has no control. A modifiable risk factor is one over which a person may exercise control, such as by changing a lifestyle or personal habit or by using medication. A risk factor may operate independently or concurrently with other risk factors. The greater amount of risk factors a person possesses, the greater the incidence of coronary artery disease (CAD) and individual has. Individuals at risk should be advised to seek regular physical examinations and to engage in heart healthy behaviors to produce a deliberate effort to reduce the number and extent of risks.

Risk factors are classified by the degree to which they can be modified by change in lifestyle or personal behaviors. Some nonmodifiable risk factors include family history of CAD (first degree relative with cardiovascular disease at 55 years of age or younger for men and at 65 years of age or younger for women), increasing age (more than 45 years of age for men, more than 55 years of age for women), gender (men develop CAD at an earlier age than women), and race (higher incidence of heart disease in African Americans than Caucasians). Modifiable risks include hyperlipidemia, cigarette smoking, tobacco use, hypertension, diabetes, metabolic syndrome, obesity, and physical inactivity. The Framingham Risk Calculator is an instrument that healthcare providers commonly utilize to estimate an individual's risk for having a cardiac event within the next ten (10) years. The application of risk calculator such as the Framingham risk score generally take into account traditional risk factors such as age, sex, blood pressure, smoking status, total cholesterol and highdensity lipoprotein cholesterol levels, and the presence of diabetes which are recommended to be used in clinical practice to estimate patients' cardiovascular disease (CVD) risk [5]

There are structural differences between the hearts of a male and female. These differences have significant implications in healthcare. It is well know that the heart of a female tends to be smaller than that of a male and the coronary arteries of a female are also narrower in diameter than a male's. Al though not everything is against a woman. Women tend to develop CHD later than a man due to the cardio protective benefits of the female hormone estrogen. For females there are three key elements of this female hormone that benefit cardiovascular health. Estrogen increases high density lipoprotein (HDL) that transports cholesterol out of the arteries, it reduces low density lipoprotein (LDL) that deposits cholesterol within the arteries, and it dilates the blood vessel which will improve blood flow to the heart.

According to Chobanian, Bakris, \& Black, et al. (2003) hypertension, mainly an elevated systolic blood pressure, increases an individual's risk of CHD in those 50 years of age and older [6]. Older patients need to be education and assisted to begin treatment with lifestyle modifications. If medications are introduced, it is recommended that the use of a diuretic be used as first or second line therapy. Older adults have multiple comorbidities and drug interaction should be at the forefront of consideration.

The Centers for Disease Control and Prevention reported in 2015, about 15 of every 100 U.S. adults aged 18 years or older $(15.1 \%)$ currently smoked cigarettes [7]. This means an estimated 36.5 million adults in the United States currently smoke cigarettes. Smoking cessation education is the single most cost effective intervention for healthcare providers to institute. Healthcare providers are key to supporting smoking cessation and educating patients on the value of such.

Diabetes mellitus (DM) is also a known to hasten the development of CHD. An increased amount of glucose in the blood stream promotes dyslipidemia, increased platelet aggregation, and altered red blood cell formation, which all can lead to formation of a thrombus. Strict control and management of patients with DM is essential to management of CHD. Those individuals who have DM may have typical or atypical signs and symptoms of acute coronary syndromes, but have increased ischemic heart disease risk.

Additionally, a patient needs to undertake a structured program of activity, exercise, and dietary modifications to reduce CHD risk factors. A diet that is low in saturated fat and high in soluble fiber is recommended. Individuals may need other specific modifications in diet, such as the patient with DM. The healthcare provider may consult a dietician to assist an individual in follow the appropriate dietary recommendations for their specific needs. In order to maintain compliance with recommendations, a patient needs to be accepting and aware of the dietary program. An introduction or maintenance of regular physical activity is essential. The healthcare team supports the patient in setting realistic goals for physical activity. Healthcare providers can instruct patients to engage in activities that interest them to maintain incentive. The healthcare provider additionally needs to educate patients on exercising to an intensity that does not impede their ability.

The healthcare community has a duty to educate the patient population on their modifiable risk factors for heart disease. Patients who perceive their modifiable risk factors and understand that they have influence in changing these risk factors maybe more likely to alter those behaviors in a positive manner. Compliance in any health care regime requires the team work of the provider and the patient in order to come to a balance that both can agree on. 
Pathophysiology

All muscles in the body require a constant blood supply. Coronary arteries that supply the myocardium stenos, or become narrowed, in acute coronary syndrome. The heart muscle then receives an inadequate blood supply because of the fat and cholesterol that subsequently narrows or clogs the coronary arteries. When one of these atherosclerotic plaques ruptures, fissures, or ulcerates it can cause a partial and/or occlusive thrombus disrupting blood flow to the muscle.

Clinical manifestations of CAD produce symptoms and complications arising from the location and degree of the narrowing of the arterial lumen, thrombus formation, and obstruction of blood flow to the myocardium, or heart muscle. This obstruction of blood flow is usually progressive, causing an insufficient blood supply to the myocardium depriving oxygen that is required for its survival. If this lack or decrease in blood supply is great enough and/or of long enough duration permanent damage and death to the myocardial cells occurs. Over time, this damaged myocardial tissue undergoes a deterioration and will be replaced by scar tissue, which causes varying amounts of myocardial dysfunction. Results may include persistent low cardiac output and heart failure where the body's need for blood supply cannot be maintained. This decrease in blood supply may even give rise to an abrupt cessation of the heart known as sudden cardiac death.

Unfortunately, individuals with myocardial ischemia may present with a range of symptoms other than chest pain. Heart disease in women develops differently than it does in men, and often progresses over a much longer period of time. The symptoms of heart disease in women may be more subtle. For this reason women tend to attribute their symptoms to some other cause. It is important for all women to be aware of the possible differences in symptoms depending on if they are male or female. Prodromal symptoms may manifest or a major cardiac event may be the first evidence of coronary atherosclerosis.

Women's symptoms angina (chest pain) is often described as a tightness in the chest, which at times radiates down the left arm or into the jaw. Additionally chronic breathlessness or waking up finding it difficult to catch one's breathe. Overwhelming and unusual fatigue is associated with heart disease in women. Dizziness, unexplained lightheadedness and blackouts are also signs that women with heart disease experience. Edema, particularly in the lower legs and ankles, profuse sweating, and vague abdominal discomfort, which may include nausea or vomiting.

On the flip side if you compare how angina (chest pain) in men as often described you can note the differences. Men describe their pain as sudden pressure, fullness, squeezing, or pain in the center of the chest that lasts more than a few minutes or goes away and comes back. Pain radiates from the center of the chest to the shoulder, neck, or arms. Chest discomfort may be accompanied with fainting, lightheadedness, profuse sweating, nausea or shortness of breath. A sudden onset of a rapid heartbeat may also be noted with an impending sense of doom.

Further complicating the presentation of cardiac events is the age of an individual. Typical pain for a male or a female decreases with age resulting in a delay of treatment. Older adults commonly present with dyspnea (shortness of breath), but less commonly with precordial pain due to decrease in pain perception. This makes them more prone to painless acute coronary syndromes. Additionally, neurological symptoms (confusion/weakness) and worsening heart failure are common in the elderly population. Co morbidities are also common in the older adult making diagnosis difficult and EKG findings are atypical.

The healthcare provider must remember that the older adult, who typically has multiple co morbidities hasan increased risk of a silent myocardial infarction. Increasing a healthcare provider's knowledge on the atypical signs and symptoms of CHD presentation in a woman and the older adult populations can increase treatment time and outcomes [8].

\section{References}

1. Mozaffarian D, Benjamin EJ, et al. (2015) Heart disease and stroke statistics - 2015 update: A report from The American Heart Association. Circulation 131: e29-322.

2. Burell G, Granlund B (2002) Women's hearts need special treatment. International Journal of Behavioral Medicine 9: 228-242.

3. The Woman's Heart Foundation (2007) Heart Attack Symptoms: An Action Plan for Women.

4. Heron M (2012) Deaths: Leading causes for 2008[PDF2.7M]. National vital statistics reports 60 .

5. Arenault BJ, Pibarot P, Despres JP (2009) The quest for the optimal assessment of global cardiovascular risk: Are traditional risk factors and metabolic syndrome partners in crime. Circulation 113: 35-49.

6. Chobanian AV, Bakris GL, Black HR, et al. (2003) Seventh Report of the Joint National Committee on Prevention, Detection, Evaluation, and Treatment of High Blood Pressure. Hypertension 42: 1206-1252.

7. Centers for Disease Control and Prevention (2016) Cigarette smoking among adults - United States, 2005-2015. Morbidity and Mortality Weekly Report 65: 1205-1211.

8. Ornato J, Hand M (2001) Warning Signs of a Heart Attack. Cardiology Patient Page. Circulation. Cross Mark 104: 12121213.

Copyright: @2017 LoriAnn F. Pajalich. This is an open-access article distributed under the terms of the Creative Commons Attribution License, which permits unrestricted use, distribution, and reproduction in any medium, provided the original author and source are credited. 